\title{
An electrophysiological method for the study of binocular rivalry'
}

WILLIAM R. BIERSDORF, DEPARTMENT OF OPTHALMOLOGY, INSTITUTE FOR RESEARCH IN VISION, OHIO STATE UNIVERSITY, Columbus, Ohio AND TED LAWWILL, DEPARTMENT OF OPTHALMOLOGY, UNIVERSITY OF LOUISVILLE, Louisville, Kentucky

A technique is described for recording cortical evoked responses from human Ss in binocular rivaly. Each target is illuminated with flickering light at different frequencies upon a steady background. While the $S$ continuously indicates with a response key which target he is seeing, the cortical responses are tape recorded. The tape recording is later analyzed b' average response computer separately for each eye, seeing and not seeing. This simultaneous record of the functioning of both eyes eliminates confounding variables found in earlier methods. Controls are included for rivaly changes during a stimulus cycle and for $S$ motor response time.

Binocular rivalry refers to the stimulus situation where dissimilar visual targets are presented to the two eyes such that they cannot be perceived as one. The observer perceives alternately the monocular targets or perceives a composite of elements from the monocular targets. The monocular elements which are not seen are described as suppressed. Interest in this phenomenon has been renewed recently because of its relevance to the suppression theory of fusion, which usually states that a similar phenomenon occurs in normal binocular vision (Asher, 1953; Hochberg, 1964).

The physiological mechanisms underlying suppression and binocular fusion are still unknown. As the phenomenon involves the simultaneous functioning of both eyes, investigators have turned their interest to the higher visual centers. A beginning toward the analysis of the problem was provided by coding the stimulus to one eye by flickering light and analyzing the EEG (electroencephalogram) produced by the flickering light in binocular rivalry (Lansing, 1964). A tuned amplifier was used in this first study to amplify the very small brain potentials produced by the flickering light. A further improvement was provided by the use of an average response computer to analyze the brain responses. enabling study of the waveform of the evoked responses as well as its average amplitude (Kaufman et al, 1965: Riggs \& Whittle, 1967: van Balen, 1964).

Certain difficulties in the design of these experiments persisted, however. Analysis of the perceiving phase and the suppression phase of rivalry were performed in sequential experimental runs on one eye instead of simultaneously on both eyes. Coding of only one eye's stimulation, instead of both, was used so that the response to the other eye was neglected. Another difficulty is presented by reports that the visual evoked response is dependent on the attention which the $S$ pays to the stimulus (Chapman \& Bragdon, 1964). Thus, the effects of changes in attention or arousal over time could be confounded with suppression effects in the sequential investigation of binocular rivalry. The present paper outlines a technique for simultaneous recording of the evoked responses from both eyes, coded and identified separately.

\section{METHOD}

The S views two different circular targets, one for each eye, which are superimposed (binocular rivalry). In the present design gratings oriented at $90 \mathrm{deg}$ to each other are presented to the two eyes. Each grating (light bars) is illuminated at a steady level of 3 $\mathrm{ft}-\mathrm{L}$ to which is added an additional illumination of $6 \mathrm{ft}-\mathrm{L}$ during the light phase of the flicker stimulation. A slow frequency of flicker stimulation (approx. $1 \mathrm{~Hz}$ ) is used to allow the long latency components of the evoked response to be recorded. With a light-dark ratio of $1: 1$ used for the flickering stimulation, the low steady illumination maintains vision of the grating during the off-phase of the flicker. Flicker stimulation is applied at slightly different frequencies in the two eyes (e.g., 0.90 and $0.95 \mathrm{~Hz}$ ). (If high frequencies of stimulation are used, the low steady illuminations of the gratings are not required.) In the present design the targets are $3 \mathrm{deg}$ in diameter surrounded by an illuminated surface of $15 \mathrm{deg}$ diameter at $0.01 \mathrm{ft}-\mathrm{L}$. This pattern is used to emphasize the photopic components of the evoked response.

The $\mathbf{S}$ views the targets in binocular superimposition and indicates continuously with a three position switch whether he is seeing bars upward to the left, bars upward to the right, or is uncertain. Standard scalp electrode placements (e.g., mid-line occipital and parietal bipolar with ear ground) were used for obtaining the evoked cortical responses. The S's switch response, the evoked cortical response, and the two stimulus waveforms are recorded on a multi-channel FM tape recorder. The tape recording is then analyzed by an average response computer synchronized first with one eye's stimulation frequency and later with the other eye's frequency. For each eye frequency the tape is analyzed separately for the dominant position and the suppressed position of the $\mathrm{S}$ switch. Provision is made in the analysis to compensate for the motor response time of the $S$ in pressing the switch, and to average only those evoked responses in which the S's switch is in one position for the entire stimulus on-cycle. The latter is required because at the slow frequencies of stimulation (about $1 \mathrm{~Hz}$ ) the S's rivalry sometimes changes during the cycle.

Stimulus Apparatus

The visual targets are presented on a Wheatstone stereoscope (Fig. 1) with arms rotating about the centers of rotation of each eye. The convergence of the arms and the interpupillary distance

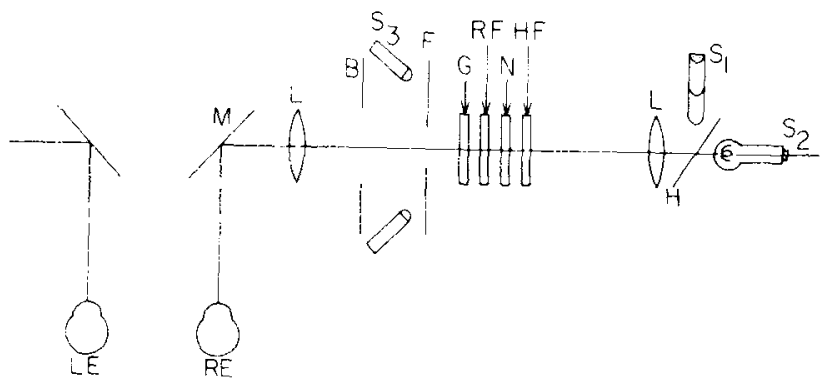

Fig. 1. Diagram of stimulus apparatus. LE is left eye. RE is right eye. Left arm of stereoscope same as right arm. Description in text. 


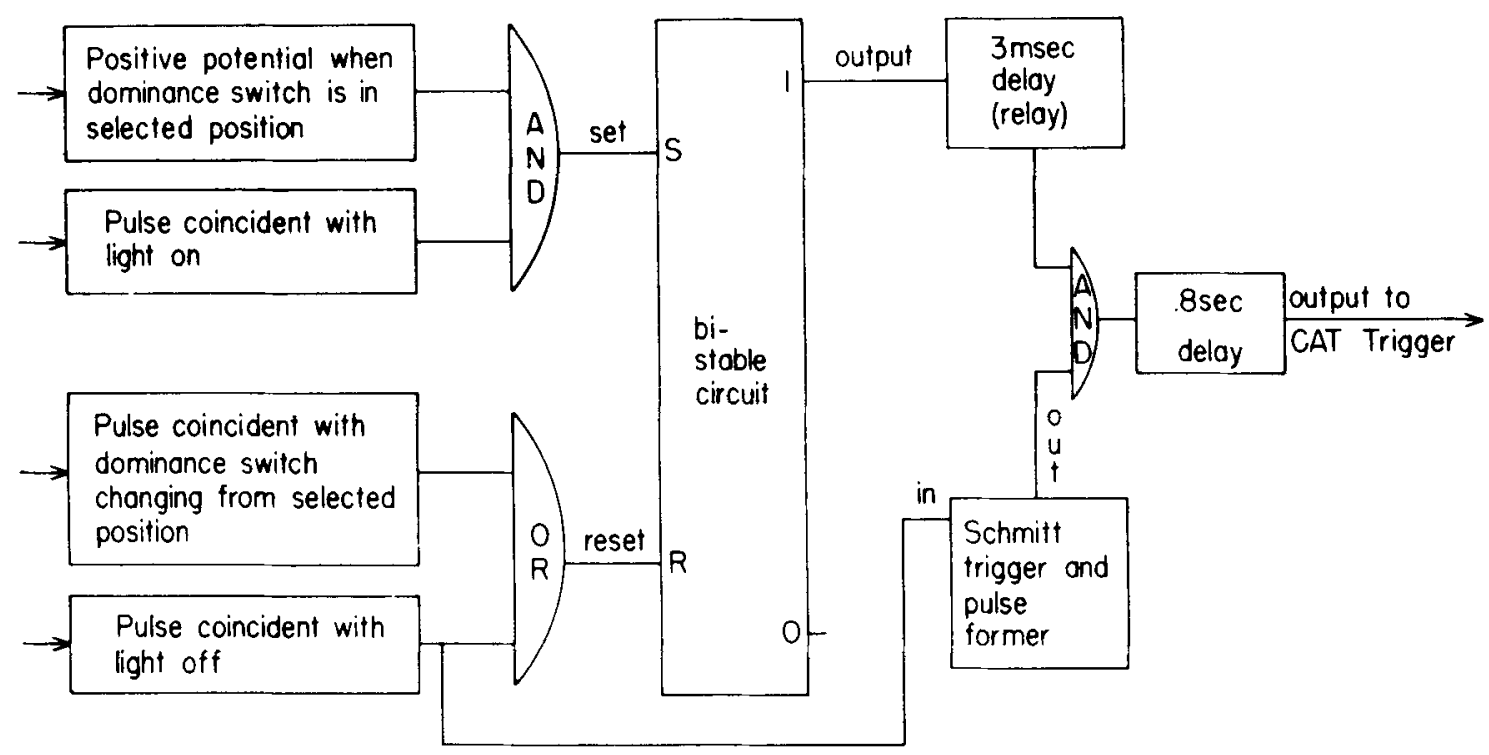

Fig. 2. Diagram of logic circuitry to control evoked response averaging. Explanation in text.

are adjusted for each $\mathrm{S}$. On each arm, continuous illumination is provided by a $6 \mathrm{~V}$ tungsten bulb $\left(\mathrm{S}_{2}\right)$ shining through a half-silvered mirror $(\mathrm{H})$. Reflected coaxially from this mirror is a second beam from a glow modulator gas discharge tube $\left(S_{1}\right)$. Both beams are collimated by a lens (L), pass through a heat absorbing filter (HF), red filter (RF, Corning 2424), and neutral density filter $(\mathrm{N})$ when needed. Each beam also passes through a field stop (F) and grating slide $(G)$ containing alternate opaque and transparent bars (each $0.5 \mathrm{deg}$ in width). The images of the filament of the tungsten bulb and the gas discharge light are focussed at the pupil by a final lens (L) after reflection from the mirror (M). With this Maxwellian view arrangement the bar pattern is in focus at the retina. Use of a Maxwellian view increases the retinai illuminance provided by the stimulus lights. Illumination of the field stop (F) painted white, is by small tungsten bulbs $S_{3}$. A stop (B) limits the extent of the illuminated surround. The head and eye positions of the $S$ are maintained with a biting board and forehead rest.

In the present apparatus, the glow modulator tubes (R1150B) have been driven by square wave (on or off) stimulation. This was provided by a sine wave oscillator feeding into a Schmitt trigger square wave device and thence to a high voltage dc amplifier. This provides square wave modulation with equal on and off times. The glow modulator tubes may also be driven by standard laboratory square wave stimulators if the output voltage is sufficient.

\section{Recording Apparatus}

The S's response switch consists of two microswitches mounted back-to-back on a light-weight lever. These switches control batteries for putting a plus or minus dc signal on tape to control the averaging. The cortical responses are amplified about 50,000 and recorded at $3-3 / 4 \mathrm{in} . / \mathrm{sec}$ on a seven channel FM tape recorder.

To compensate for the motor response time of the $\mathrm{S}$ after the perceived change in rivalry, the cortical response record is delayed with respect to the $S$ 's perceptual response and the stimulus waveforms. The cortical evoked response, $S$ switch response, and the two stimulus waveforms are recorded on four channels of the tape together with an audio channel for experimental identifica- tion. At the same time, the evoked response is retrieved from the playback head and fed back to another recording head on the same tape. From the spacing of the heads, the second evoked response channel is thus delayed $1.43 \mathrm{sec}$ from the rest of the tape signals. The computer trigger pulse is delayed $0.8 \mathrm{sec}$, before which time the decision is made by the digital logic circuitry whether to average that response. If the $S$ switch is in the correct dominance position for the entire on-cycle of the stimulus, the tape is then averaged from the delayed evoked response channel with the computer synchronized with the onset of the stimulus for that eye.

The decision making apparatus is illustrated in Fig. 2. The key element is the Schmitt trigger and pulse former which produces a pulse synchronous with the selected (right or left) light stimulus going off. The rest of the apparatus is used to gate the pulse from the pulse former. The rest consists of a bistable circuit with the "set" fed by an AND gate and the "reset" fed by an OR gate. The AND gate receives a positive signal level coinciding with the dominance switch being in the proper position; and it receives a pulse coinciding with the onset of the light stimulus. The iwo light stimuli levels feed into a selector switch followed by a capacitor and diode to provide the onset and offset light pulses. Thus, the S's dominance switch has to be in the proper position before the stimulus onset can set the bistable circuit. The OR gate rese ts the bistable circuit when the S's switch changes position or the stimulus light goes off. The output of the bistable circuit, when set, energizes a fast action relay which gates the pulse former. The pulse former gives a pulse when the light stimulus goes off. If the bistable circuit stays set until rese thy the stimulus going off, the $3 \mathrm{msec}$ delay of the gating relay opening is just long enough to let the pulse from the pulse former through before the relay opens. This pulse is then delayed $800 \mathrm{msec}$ by a Tektronix pulse generator and waveform generator before triggering the averaging computer. This delay plus the length of the stimulus on-time subtracted from the $1.43 \mathrm{sec}$ delay of the cortical response channel means that the computer sweep begins about $130 \mathrm{msec}$ before the stimulus onset time on the cortical channel. This is the compensation for the motor response time of the $S$ after perceiving a rivalry change. The computer analysis time of 
15: se thus covers appowmateis the first 370 moes of the corcical response after stimulus onser. The stimulus onset point in the records was checked by putting a photo-cell signai through the cortical resporise channel and recording it with the computer. The stimulus periods were checked and found to be consistent.

Each tape recording is analyzed four times by an average response computer (Mnemotron CAT or Fabri-Tek signal averager). The four analyses are: (1) response to the right eye stimulus when the right eye is dominant. (2) response to the right cye stimulus when the laft eye is dominant. (3) response to the left eye stimulus when the left eyc is dominant. and (4) response to the left eye stimulus when the right eye is dominant.

As the responses are averaged from the tape record of the simultaneous functioning of both eyes, the degree of control inherent in this procedure is great. If one eye is misaligned. the effective brightness of the stimulus to that eye is decreased. If this produces a smaller response when that eye is suppressing. it also produces a smaller response when the same eye is dominant. Hence the effects of misalignment or other stimulus artifacts are cancelled out and cannot account for the differences between dominant and suppressed evoked potentials from the same eye. Similarly, changes in attention or arousal over time are not confounded with rivalry changes as the responses from both eyes are recorded simultaneously.

\section{REFERENCES}

ASHI:R. H. Suppression theory of binocular vision. Brit. J. Ophthal., 1953, 37. 37.49.

(HAPMAN. R. M.. \& BR.AGDON. H. R. Froked responses to numerical and nun-numerical visual stimuli while problem solving. Noture. 1964, 20.3. $1155-1157$.

HOCHBI:RG, J. Depth perception low with local monocular suppression: A problem in the explanation of tercopsis. Science. 1964, 145, 1334-1336.

KalliMAN, L.. PITBLADO. $(. . \&$ MILLIR. J. I.. Jr. Perceptual phenomena and evoked cortical posentials resulting from binocular timulation with flickering light. Report SRRC-RR65-101 Vov. 1965. Sperry Rand Research Center. Sudbury. Mass.

LANSING. R. W. Flectroencephalographic correlatw of hinocular rivalrs in man. Science, 1964.14h.1325.132?.

RIGGS. L. A. \& WHITTLE. P. Human octipital and retinal polentials evoked by subiectively faded visual timuli. Iis. Res. 1967.7.441-451. van B.ALLN. A. T. M. The influence of uppression on the tlicker I.RG. In H. 1. Henker and L. H. van der Tweel dids.l. Flicker. The Hague: Dr. W. Junk. 1964. Pp. 440-446.

\section{NOTE}

1. This apparatus was devcloped at the Walter Reed Army Institute of Restarch and the author would like to acknowledge the tichnical assistanci of SP4 Charlo (aidwell.

\section{A runway for the fish'}

\author{
ALCINE POTTS AND M. E. BITTERMAN, DEPARTIIENT OF \\ PSYCHOLOGY, BRYN MAWR COLLEGE, Bryn Mawr, Pennsy \% \\ vania
}

A runway and a training procedure are described. Acquisition and extinction curves for a sample of 12 goldfish trained in spaced trials are plotted in terms of three measures.

The apparatus here described for the study of learning in the fish is directly analogous to the runway which has played so large a part in work with the rat. In our previous experiments on food-rewarded instrumental learning in the fish, we have used a manipulative rather than a locomotor response-nosing or biting a lever introduced into the animal's individual living tank. in which it is carried to the experimental situation (Longo \& Bitterman, 1959). The lever is, of course, more efficient in many respects and more objective than the runway, but there are kinds of experiments that can be done more conveniently with the runway than with the lever, or not at all with the lever, response to which must be shaped by special training procedures (Bitterman, 1966). The only serious difficulty that we encountered in our attempt to develop a runway for the fish stemmed from the fish's aversion to handling (or netting) and its consequent reluctance to enter a goalbox from which it has been removed by such means. Our solution to the problem of transferring the animal from one place to another is simply to scoop it up in a container of clear plastic. a procedure which minimizes its contact with injurious surfaces.

A satisfactory runway for $3-4$ in. goldfish is diagrammed in Fig. 1. It is made of $1 / 2$-in. black Plexiglas. The ends of the main pieces are milled. drilled, and tapped, then fastened together with brass screws, after which Plexiglas solvent is run over the joints. A hinged cover divided into four parts fone for the starting compartment, two for the main section, and one for the goalbox) is made of $1 / 4-i n$. diffusing Plexiglas. The inside depth of the runway. which is $3 \frac{1}{2}$ in. wide throughout, is 6 in., and it is filled to a depth of $4 \mathrm{in}$. The starting compartment, $12 \mathrm{in}$. long, is set

Fig. 1. The runway. $D_{1}$ and $D_{2}$, guillotine doors; $P$, photocell ; $\mathrm{L}$, lamps; $\mathrm{F}$, opening for base of funnel through which worms are discharged by the feeder.

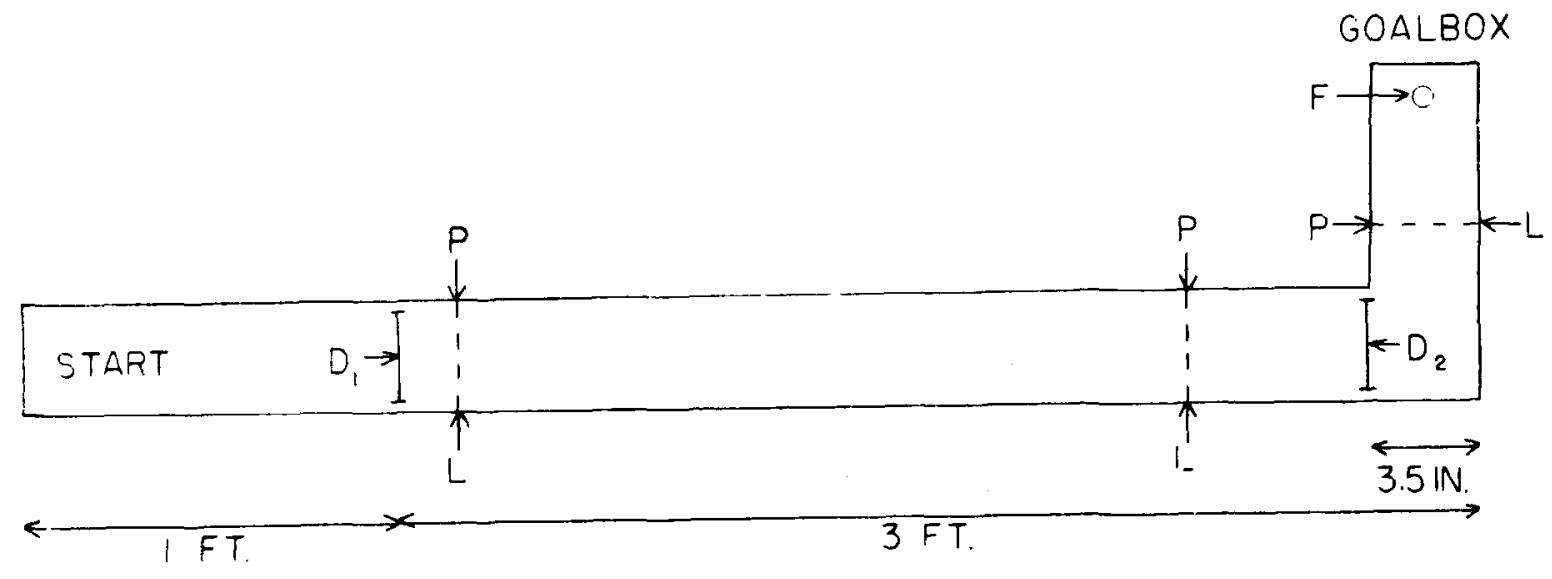

